\title{
Retos en la implementación de las TIC para el proceso de negociación internacional
}

\author{
Challenges in Implementing ICT for International Negotiation Processes \\ Défis de la mise en œuvre des TIC dans le processus de négociation internationale
}

José A. Cano Arenas

Docente Tiempo Completo, Facultad de Ciencias Económicas y Administrativas, Universidad de Medellín, Medellín - Colombia. Grupo CYGO, reconocido por COLCIENCIAS. Magíster en Ingeniería

Administrativa.

E-mail: jacano@udem.edu.co

José J. Baena Rojas

Docente Tiempo Completo, Facultad de Ciencias Económicas y Administrativas, Universidad de Medellín, Medellín - Colombia.

Grupo GINI, reconcido por COLCIENCIAS. Maestría Oficial en Internacionalización.

E-mail: jjbaena@udem.edu.co

Artículo de investigación científica y tecnológica Según clasificación COLCIENCIAS

Recepción: 01/06/2013

Corrección: $15 / 10 / 2013$

Aprobación: 14/11/2013

\section{Resumen}

El propósito de este artículo científico es identificar los factores que influyen en la adopción o rechazo de las tecnologías de información y comunicación (TIC) para la negociación en procesos de compraventa internacional. Se realizó un estudio en 180 empresas de la ciudad de Medellín, Colombia, dedicadas a actividades de importación y/o exportación, donde a través de entrevistas telefónicas y análisis cuantitativos y descriptivos se evaluaron las desventajas en el uso de las TIC, las preferencias en la manera de negociar (cara a cara o con las TIC), elementos propios en el mejoramiento de las relaciones y aspectos que afectan la implementación de las TIC especializadas para la negociación. El estudio muestra que los mayores impedimentos para implementar las TIC especializadas en la negociación se deben al desconocimiento en sí de ellas, el no reconocer la necesidad de las TIC en las empresas, y el costo asociado con la adquisición y buen funcionamiento de estas tecnologías. También se concluye que las TIC para la negociación internacional deben permitir el alcance de objetivos de forma efectiva, generar confianza y un ambiente propicio para el mantenimiento de relaciones entre los negociadores.

Palabras clave: compraventa internacional, empresas, negociación internacional, tecnologías de información y comunicación, TIC.

\section{Abstract}

This scientific article sought to identify factors influencing the adoption or rejection of information communication technologies (ICT) for international negotiation of sales processes. The study focused on 180 companies in the city of Medellin, Colombia, engaged in import and/ or export activities; these companies were evaluated on the disadvantages of using ICT, preferences on how to negotiate (face to face or ICTmediated), elements to improve relations and issues affecting the implementation of specialized ICT for negotiation. The study shows that the biggest impediments to implementing specialized ICT for negotiation are due to lack of knowledge of the ICT itself, failure of companies to recognize the need for ICT, and the costs associated with the acquisition and operation of these technologies. It was also concluded that ICT for international negotiation should allow effective achievement of goals, build trust, and an appropriate environment to maintain relationships among negotiators.

Keywords: enterprise, international negotiation, information and communication technologies, ICT, international sale.

\section{Résumée}

Ĺobjectif de cet article scientifique est d’identifier les facteurs qui influent sur l’adoption ou le refus des technologies de l’information et de la communication (TIC) pour la négociation dans les processus d'achat-vente internationale. On a réalisé une étude dans la ville de Medellín, en Colombie, sur 180 entreprises qui développent des activités d`importation et/ou d’exportation. À partir d`interviews téléphoniques et des analyses quantitatives et descriptives, on a évalué les désavantages dans l'usage des TIC, les préférences dans la façon de négocier (face à face ou avec les TIC), ce qui constitue des éléments propres du processus d'amélioration des relations et des aspects qui affectent directement la mise en œuvre des TIC spécialisées pour la négociation. Ĺétude montre que la plupart de troubles pour la mise en place des TIC spécialisées en négociation sont à cause de la méconnaissance de celles-ci ; à la non reconnaissance du besoin des TIC dans les entreprises ; et au coût qui implique l'acquisition et le correct fonctionnement de ces technologies. On conclut aussi que les TIC pour la négociation internationale doivent permettre la réussite des objectifs de façon efficace, et de développer de la confiance et une ambiance propice pour garantir de bonnes relations entre les négociateurs.

Mots clef: achat-vente internationale, entreprises, négociation internationale, technologies de l'information et de la communication TIC. 


\section{Introducción}

El presente artículo científico pretende identificar en términos generales, algunos de los factores que pueden incidir en la adopción o rechazo de las tecnologías de la información y la comunicación (TIC) dentro del proceso de negociación en las operaciones de compraventa internacional de las empresas de la ciudad de Medellín, las cuales en efecto han logrado internacionalizarse, ya sea efectuando exportaciones o incluso importaciones.

También se busca determinar algunas de las causas por las cuales las empresas consideran que las TIC pueden ayudar al mejoramiento de las relaciones en la negociación dentro para los procesos de compraventa internacional en la ciudad de Medellín. Paralelamente es necesario establecer algunas de las desventajas que perciben las empresas (exportadores e importadores) a partir del uso de las TIC en la negociación para los procesos de compraventa internacional y finalmente describir las causas por las cuales las empresas (exportadores e importadores) no se motivan en su mayoría a adoptar en la negociación las TIC especializadas para la compraventa internacional.

Partiendo entonces de la premisa de que la negociación y los procesos de compraventa internacional hacen un uso cada vez más intensivo de este tipo de herramientas, este artículo apunta a identificar algunos de los motivos por los cuales algunas empresas que han logrado internacionalizarse, tienen aún dificultades para implementar estas herramientas pese a todos sus beneficios, los cuales tienden a favorecer no solo la materialización del intercambio, sino que además tienden a generar que este se efectúe de forma eficiente, lo que termina redundando en la optimización de muchos de los procesos mismos de las empresas, todo ello siempre y cuando se lleve a cabo más inversión por parte de las empresas en la adquisición misma de este tipo de tecnologías (FernándezMenéndez, López-Sánchez, Rodríguez-Duarte y Sandulli, 2009).

Es así entonces como las TIC constituyen el eje central del presente artículo, en especial cuando son este tipo de herramientas las que pueden incidir favorablemente en los procesos de negociación y por ende en la consecución misma de intercambios entre empresas; de este modo se intenta determinar concretamente en ciertas empresas de Medellín, cuáles son las dificultades más sobresalientes por las cuales estas no logran hacer uso de las TIC de manera más intensiva dentro de sus procesos de negociación, pese a los diversos beneficios que parecen generarse por su implementación.

Por lo tanto, si los efectos de las TIC pueden influir en el comportamiento de las empresas no solo en el crecimiento de las ventas sino en la estandarización misma de sus procesos y sucesivamente en la optimización de sus procesos, resulta imperativo analizar dentro de la negociación qué retos y cuáles aspectos se pueden considerar claves para que las organizaciones adopten no solo las TIC sino que hagan una selección idónea de estas herramientas en las operaciones de compraventa internacional, a fin de alcanzar lo que denominan (Bayo-Moriones, Billon, y Lera-López (2013) “innovación organizativa”. No obstante, para lograr este propósito tal y como los mismo autores señalan, es necesario implementar prácticas de trabajo integrando mejoras para la comunicación interna y externa en la empresas, también establecer una selección apropiada de herramientas, lo que en algunos casos puede tomar años desde la implementación misma de todas estas prácticas de trabajo; y este es uno de los motivos por los cuales las empresas pueden llegar a dejar de lado las TIC porque en algunos casos estas desconocen su funcionamiento, su versatilidad e incluso porque desconocen sus beneficios en el tiempo; así, todas estas herramientas puedan terminar redundando positivamente en una mayor cuota de mercado y un mejor margen de ganancias De otro lado es importante mencionar cómo aparte del tiempo y el nivel de preparación de la empresa misma para insertarse a este paradigma, existen otros factores que evidentemente pueden incidir también en la implementación de las TIC en el proceso de la negociación, tal y como sucede con aspectos propios de la percepción, además de otros elementos emocionales en torno a la ética, los cuales en efecto pueden intervenir entre las partes que participan en la negociación. Así en este orden de ideas, si bien las TIC pueden incidir en el aumento de los intercambio de las empresas, paralelamente estas herramientas también pueden interferir desfavorablemente en tales intercambios, todo ello en la medida de que las partes lleguen a percibir algún tipo de ausencia en la confianza, lo que en definitiva tiende a generar distorsiones en la construcción misma de las relaciones comerciales y por ende en el entramado de la negociación (Yang, Chandlrees, Lin y Chao, 2009) o incluso como bien puede adaptarse en este caso concreto para las operaciones de compraventa internacional, por los inconvenientes asociados con la credibilidad y seguridad misma que este tipo de transacción comercial requiere.

Por consiguiente, teniendo en cuenta todas estas nociones teóricas es evidente que existen factores que inciden en la no implementación de estas herramientas, supeditadas en primer lugar a las desventajas en el uso de las TIC, las cuales pueden estar asociadas por ejemplo con el tiempo que pueden tardar las empresas en el proceso de selección de la herramienta más apropiada según sus propias necesidades y capacidades. En segundo lugar, supeditada a las preferencias en la manera de negociar, en cuyo caso pueden intervenir aspectos culturales, éticos y sociales los cuales tienden a generar que las empresas no perciban las TIC como propiamente una herramienta; y en tercer y último lugar, supeditada al mejoramiento de las relaciones, en donde la construcción de vínculos comerciales que permitan el afianzamiento de la confianza entre las partes, pueden para las empresas no estar asociadas con el uso de las TIC porque durante mucho tiempo los procesos de negociación y las operaciones de compraventa internacional no hacían propiamente un uso intensivo de estas herramientas.

En consecuencia el presente artículo ahonda respecto a estas tres categorizaciones para establecer posibles puntos de partida, respecto al porqué las empresas de 
la ciudad de Medellín -tenidas en cuenta como objeto de estudio- aún tienden a no ser completamente receptivas en la implementación de TIC en la negociación como una herramienta para dinamizar las operaciones de compraventa internacional (exportación y/o importación). También la investigación a través de un análisis cuantitativo y descriptivo exalta todas aquellas dificultades que hacen que en las empresas de la ciudad de Medellín aún no se implemente en su totalidad las TIC y en ocasiones prefieran la negociación cara a cara, y de igual forma establece una noción general de cómo estas tecnologías pueden mejorar relaciones en la negociación internacional.

Finalmente, dentro del artículo se establecen algunas conclusiones en torno al uso de las TIC en la negociación y sobre como existen aún diversos aspectos que deben ser tenidos en cuenta, ya que muchas empresas no logran implementar aún dentro de sus operaciones de compraventa internacional estas herramientas, pese a todos sus beneficios y la multiplicidad de ventajas, en cuyo caso se pueden destacar sistemas más eficaces para el rastreo y la captura de información valiosa de los clientes finales, como ciertos portales especializados en la red (Albors-Garrigos, Hervas-Oliver y Márquez, 2009).

\section{Marco teórico}

\section{La negociación internacional y las TIC}

En un proceso de negociación es evidente que existe un escenario en cuyo caso las partes irrefutablemente buscan satisfacer sus necesidades a partir de las concesiones que estas mismas puedan otorgar, en procura de materializar la transacción, pero aparte de este principio lógico y obvio, también se torna imprescindible encontrar alternativas que permitan la consecución eficiente de todo este proceso.

Es entonces desde esta lógica que se construye la polémica sobre qué es más conveniente y mejor en una negociación, particularmente en un proceso de compraventa internacional en donde las partes en un primer momento no se conocen y en donde la construcción de confianza-al parecer-tiene su origen cuando las partes interactúan y conocen a fondo la identidad no solo de la empresa, sino de algunos aspectos personales de quien, como contraparte, interviene en la negociación. Es desde tal lógica cuando el concepto estilo de negociación se hace evidente, debido a que los patrones propios de conducta de las empresas y sus representantes, respecto al cómo se pactan las transacciones no es precisamente igual; este con el tiempo y con las tendencias mismas de las TIC ha cambiado, manifestándose así nuevas oportunidades dada la naturaleza ubicua que ofrecen estas herramientas.

No obstante y pese a las ventajas que pueden ofrecer las TIC en torno a cómo se puede administrar la información, la distancia y el tiempo de las partes que intervienen en la negociación, aún existe una vehemente dicotomía entre si, es más conveniente una negociación cara a cara o una negociación mediada con las TIC (Pauleen y Yoong, 2001).
Pero indiferente de esta situación, es cierto que existe una serie de factores que inciden en la no implementación de las TIC, los cuales se pueden categorizar en tres grupos y que explican en muy buena medida por qué las empresas a nivel general pueden tornarse reacias a hacer uso de todas estas herramientas dentro de los procesos de negociación y sucesivamente en las operaciones de compraventa internacional.

En primer lugar, las desventajas en el uso de las TIC, que pueden explicar en buena parte por qué existen muchas empresas que se resisten a implementar este tipo de herramientas no solo dentro de los procesos internos de la empresa sino durante la negociación. Así, resulta coherente agregar que las empresas en muchos casos toman tiempo estableciendo cuál es el mejor modelo para direccionar de manera efectiva muchos de los proceso internos que se llevan a cabo dentro de estas y es precisamente todo esta situación la que se suele denominar como innovación organizativa; sin embargo, aunque existe la evidencia empírica que demuestra una relación positiva entre la adopción de las TIC y todas las medidas de rendimiento percibido, el impacto no siempre es inmediato porque los efectos de retardo y duración difieren según el tipo de TIC que se implemente dentro de las empresas además de que debe existir una necesidad de persistencia en el uso de estas herramientas todo ello con el fin de obtener buenos resultados en algunas áreas de la organización (Bayo-Moriones et al., 2013) como bien podría ser además el caso de la negociación y el proceso de compraventa internacional. Incluso es posible afirmar que estudios previos como los de Hambrick (2007) sostienen que el comportamiento de las organizaciones tienen que ver con el conocimiento de sus dirigentes y las decisiones de sus administrativos desde el ámbito de sus características personales e incluso valores.

Así, todo este aspecto se puede resumir a las dificultades a nivel general que las empresas suelen experimentar para adaptar dentro de todos sus procesos internos el uso mismo de las TIC, porque de por medio pueden estar variables como los costos, la adopción de nuevas infraestructuras, la velocidad de las redes que garanticen el funcionamiento de la internet, el hardware y por supuesto la capacitación del personal para que hagan uso cabal y efectivo de todas estas herramientas y se alcance en definitiva un mejoramiento real en la dinámica de toda la empresa.

Con respecto a este punto es claro que para nuevas empresas, empresas pequeñas y empresas con aversión al riesgo, se sugiere utilizar estrategias pasivas de "esperar y mirar", en la cual se recomienda observar experiencias de otras empresas al implementar las TIC en sus procesos; para lograr esta forma evitar el riesgo de invertir recursos que no aportarán nada. Para empresas grandes y bien establecidas se sugiere adoptar estrategias fuertes, en especial si se desea ser líder del mercado. Esta estrategia vale la pena implementarla si se esperan unos beneficios mayores a los riesgos que representa la adopción de las TIC (Mibenge y Ngozika-Chinyere, 2007). También, aunque muchas 
TIC puedan representar altos beneficios para el proceso de negociación, se recomienda tener precaución en su implementación y asegurar que esto se haga en el momento y condiciones correctas. Además, no todas las TIC son adecuadas para todo tipo de empresa, y su elección depende de las condiciones que tengan el comprador y vendedor, tales como el tipo de negocio, del nivel de desarrollo tecnológico, cultural y económico, entre otras cosas (Mibenge y NgozikaChinyere, 2007).

En segundo lugar, las preferencias en la manera de negociar, pueden también explicar en buena medida a la manera de negociar o incluso al mismo estilo de la negociación en donde las partes deben establecer hasta cierto punto concesiones que permitan favorecer el intercambio de la compraventa internacional, como bien pueden ser la implementación misma de las TIC. Llamativamente dentro del presente estudio en muchos casos las empresas de Medellín experimentan una notable dificultad explicada por el cómo la contraparte no suele aceptar el uso de estas herramientas.

Particularmente toda esta situación en términos generales desde diversos estudios como los de Yang, Chandlrees, Lin y Chao (2009) se explica a partir del cómo intervienen diversas variables de orden cultural en donde aún existen dificultades para asimilar no solo los cambios tecnológicos particularmente por el cómo se puede tergiversar la credibilidad; es pues en este sentido que la ética se torna determinante porque en muchos casos el hecho de no interactuar de un modo más real, puede incidir en la credibilidad sobre la contraparte, de ahí que los prejuicios y otras variables sociales se tornen claves respecto al cómo se percibe el uso de la Internet, debido a la identidad no siempre comprobable de los usuarios (Chai y Kim, 2012) todo esto considerando el hecho de que es la red la que hace factible en gran parte el funcionamiento de las TIC en la actualidad dada la posibilidad de interacción en línea.

En tercer y último lugar, el mejoramiento de las relaciones, pueden explicar también cómo se construyen relaciones comerciales de corto y largo plazo; sin embargo, muchas empresas no han sido completamente dependientes de las TIC, lo que puede poner en duda la eficacia y la contundencia de estas para mejorar sus procesos internos y sobre todo la negociación en la compraventa internacional.

Para las TIC basadas en Internet y enfocadas en el abastecimiento, Mibengue y Ngozika (2007) afirman que para especialistas que trabajan en esta área existen tres preocupaciones importantes que son la seguridad, la confianza y la relación entre vendedor-comprador. Esto se hace aún más evidente en el uso de las TIC automatizadas para la negociación, debido a que se requiere compartir datos confidenciales como financieros y modelos de compras. Esto genera un reto en las organizaciones para saber qué información compartir y con cuáles otras organizaciones - contrapartes compartirla. Además de la confianza que se debe crear en las contrapartes al utilizar las TIC automatizadas para la negociación, también hay que cambiar roles y responsabilidades administrativas, lo cual incluye eliminación y creación de cargos en la empresa.

\section{Dificultades en la implementación de las TIC en la negociación internacional}

Es evidente entonces que existen asuntos de profunda discusión, debido a que la construcción de vínculos comerciales que faciliten el afianzamiento de la confianza entre las partes, pueden para las empresas no estar asociadas con el uso de las TIC; esto porque el proceso de negociación en la compraventa internacional no ha sido estrictamente dependiente del uso de este tipo de herramientas en donde la confianza es determinante dentro de la negociación; los modelos de negociación que hacen uso de las TIC, requieren de mecanismos de confianza significativamente altos, porque de estos depende el éxito dentro de las operaciones que se basan en este modelo, todo ello por la ausencia de contacto real y es esto a la que se denomina como "confianza del sistema" dentro del comercio electrónico (Pennington, Wilcox y Grover, 2003).

A continuación en la Tabla 1 llevada a cabo a partir de los estudios de Bayo-Moriones et al. (2013), Mibenge y Ngozika-Chinyere (2007), Yang et al. (2009), Chai y Kim (2012) y Pennington et al. (2003) establece desde los tres ejes del presente marco teórico algunas desventajas en el uso de las TIC en la manera de negociar y mejoramiento de las relaciones como las dificultades percibidas por la empresa a partir de tales nociones.

\begin{tabular}{|c|c|c|}
\hline Factor & Características & Dificultades percibidas \\
\hline $\begin{array}{l}\text { Desventajas en } \\
\text { el uso de las TIC }\end{array}$ & $\begin{array}{l}\text { Se suele asociar con el } \\
\text { tiempo que pueden } \\
\text { tardar las empresas en el } \\
\text { proceso de selección de } \\
\text { las herramientas más } \\
\text { apropiadas según sus } \\
\text { propias necesidades y } \\
\text { capacidades. }\end{array}$ & $\begin{array}{l}\text { * Infraestructura } \\
\text { * Hardware } \\
\text { * Velocidad de redes } \\
\text { * Capacitación de } \\
\text { persona } \\
\text { * Costo }\end{array}$ \\
\hline $\begin{array}{l}\text { Preferencias en } \\
\text { la manera de } \\
\text { negociar }\end{array}$ & $\begin{array}{l}\text { Se suele asociar con } \\
\text { preferencias en la } \\
\text { manera de negociar, en } \\
\text { cuyo caso pueden } \\
\text { intervenir aspectos } \\
\text { culturales, éticos y } \\
\text { sociales de ahí que se } \\
\text { considere importante el } \\
\text { estilo cara a cara. }\end{array}$ & $\begin{array}{l}\text { * Despersonalización } \\
\text { * Credibilidad } \\
\text { * No aceptación de la } \\
\text { contraparte de las } \\
\text { herramientas }\end{array}$ \\
\hline $\begin{array}{l}\text { Mejoramiento } \\
\text { de las relaciones }\end{array}$ & $\begin{array}{l}\text { Se asocia en cómo las } \\
\text { herramientas no } \\
\text { necesariamente son } \\
\text { percibidas como } \\
\text { imprescindibles teniendo } \\
\text { en cuenta cómo se ha } \\
\text { negociado } \\
\text { tradicionalmente. }\end{array}$ & $\begin{array}{l}\text { * Confianza } \\
\text { * Vínculos } \\
\text { * Falta de contacto } \\
\text { real }\end{array}$ \\
\hline
\end{tabular}


En resumen, existen diversas investigaciones que muestran cómo el desarrollo de relaciones dentro de los equipos virtuales, tienden a constituirse como un factor importante para relaciones de trabajo efectivas como demuestran Thompson y Nadler (2002) quienes realizan un estudio donde identifican teorías de interacción sobre el comportamiento social en medios electrónicos como por ejemplo la construcción de relaciones, difusión social, coordinación e intercambio de información y aclaran que las TIC a utilizar crean grandes diferencias en los resultados de negociación, resaltando que quienes realizan negociaciones cara a cara o mediadas por teléfono, convergen en una sola oferta de una forma más rápida que aquellos que sólo se comunican de forma escrita lo que demuestra que las herramientas TIC deben en muchos casos ser combinadas para lograr mejores resultados dentro del proceso de negociación. De hecho para solucionar problemas que suelen generarse con las negociaciones por medio de las TIC, Low y Ang (2011) sugieren que se puede combinar varias herramientas de este tipo como e-mail, teleconferencia y mensajería instantánea para intensificar las comunicaciones, crear relaciones y confianza entre los negociadores.

Aunque las negociaciones cara a cara parezcan ser mucho más confiables en términos generales que las negociaciones que hacen uso de las TIC tal y como concluyen Citera, Bearegard y Mitsuya (2005) quienes realizan una comparación de la credibilidad de negociaciones electrónicas y de negociaciones cara a cara, concluyendo que existe mayor escepticismo sobre la credibilidad de los negociadores electrónicos. Esto no significa en definitiva que las TIC no puedan convertirse en una herramienta importante para lograr modificar este patrón de conducta en el imaginario de las personas y de las empresas, y así se pueda establecer que en la actualidad este es quizás uno de los principales retos en la implementación de las TIC para el proceso de negociación internacional de empresas. Las evidencias teóricas respecto a los beneficios de las TIC son mucho más contundentes como bien se ha hecho mención, y por lo tanto, en cuestión de tiempo con nuevas medidas de seguridad posiblemente todos estos aspectos de percepción deberán cambiar, porque de ello depende la eficiencia global de desarrollo de sistemas supeditada a la confirmación de datos e información (Chang, Chen, Hung y Chang, 2012)

De otro lado se puede agregar que en la negociación llevada a cabo con las TIC se identifica también diversas ventajas porque el lenguaje que se emplea dentro de estas, se convierte en un mecanismo importante para llevar a cabo estrategias de negociación (Sokolova y Szpakowicz, 2007). Incluso en otros estudios anteriores Sokolova, Shan y Szpakowicz (2006) concluían que en ciertas condiciones, la riqueza de vocabulario, complejidad de lenguaje y predicción de texto son similares en negociaciones cara a cara y negociaciones electrónicas, lo que de nuevo parece convertirse en un importante argumento a favor de las TIC como herramienta en el proceso de negociación y en este caso concreto para las operaciones de compraventa internacional.

En diversos estudio empíricos se logran demostrar otras bondades de las TIC en la negociación pese a las dudas que se presentan en el imaginario de las empresas; por ejemplo Gallin, Gross y Gosalker (2007) hacen un ejercicio de comparación de la negociación electrónica y la negociación tradicional cara a cara, apoyándose de 80 estudiantes jóvenes que se expusieron a los dos tipos de negociación, hallando que el medio y la secuencia de negociación, escasamente afecta el resultado. En el estudio se concluye entonces que en términos de precio final, cuotas de pago y el avance en pagos, la negociación cara a cara y la negociación electrónica no presentan diferencias, sin embargo, el tipo de medio y la secuencia a usar afectan significativamente la duración de la negociación y el uso de tácticas duras o blandas.

Otros estudios (Hatta y Ken-ichi, 2008) en cuyo caso 43 estudiantes fueron puestos bajo en alguna de las cuatro condiciones siguientes: anonimato visual, no anonimato, condiciones remotas y condiciones cercanas, demuestra que en algunos casos el anonimato visual y la distancia remota inhiben la activación del patrón normal de desconocimiento de la contraparte en la negociación, lo que significa que se tiende a que los negociadores desistan de la actual negociación que están llevando a cabo. Este es otro argumento fuerte en contra de las TIC en la negociación internacional pero también se puede trabajar en la medida en que se usen las mismas herramientas para elevar la frecuencia de la comunicación lo cual claramente puede incidir en la construcción de vínculos y relaciones comerciales.

Como bien se ha señalado con antelación otros aspecto que se puede considerar es el hecho de que en muchos casos las empresas desconocen los servicios ofrecidos por las TIC, de ahí que en la mayoría de casos el correo electrónico sea la TIC más empleada no solo porque es fácil de usar, sino porque es una herramienta elemental en las empresas. En términos generales, el correo electrónico se considera un medio austero porque no es capaz de transmitir señales visuales o verbales, lo cual hace que los negociadores transmitan y reciban información de una forma distinta a como lo harían cara a cara. La falta de señales visuales y verbales reducen la presencia social de la contraparte y aumentan la distancia percibida entre estas partes (Ebner, Bhappu, Gerarda, Brown, Kovach y Schneider, 2009). Sin embargo, los estilos de comunicación utilizados en correo electrónico se centran más en tareas específicas de la negociación (ir al grano, manos a la obra) y menos concentradas en la construcción de relaciones, y obliga a que la comunicación sea asincrónica, donde cada parte debe esperar su turno para comunicarse y así se previene la interrupción de los participantes en la comunicación, 
dando la oportunidad de que cada parte exprese sus puntos de vista completamente (Ebner et al., 2009) además de que los aspectos de confianza que también se han mencionado antes, pueden ser superados con la combinación de otras TIC, además, como previamente se mencionó, existen TIC muy específicas para tareas y operaciones concretas de la negociación (Bayo-Moriones et al., 2013).

En las negociaciones cara a cara es más frecuente que se intercambie más información entre personas debido a que interactúan más por la cercanía física que tienen y por la riqueza que puede tener la comunicación visual y verbal. Además, cara a cara se intercambia información más rápida de lo que de forma escrita se haría, y prácticamente en una sesión se alcanzan los objetivos propuestos para una comunicación, mientras que por otros medios asincrónicos esto puede tomar varias horas, días e incluso semanas. Sin embargo, las negociaciones cara a cara abren la posibilidad también de que se toquen temas que no son relevantes para el proceso de negociación, resultante esto en discusiones y negociaciones menos eficientes (Thompson y Nadler, 2002).

Finalmente Low y Ang (2011) realizaron un estudio cuya muestra fue de 61 gerentes o líderes de empresas y académicos universitarios, los cuales utilizaron las TIC para las negociaciones diarias de sus compañías. De esto obtuvieron que los negociadores ( $87 \%$ ) suelen comprar a personas con las cuales se sienten cómodos y con quienes se sienten mejor para relacionarse; para el $89 \%$ de los gerentes las negociaciones electrónicas son impersonales, y esto hace que mucha información importante en la negociación se omita debido a la falta de comunicación formal y falta de oportunidades de aclarar cosas y negociar. Se dificulta crear buenas relaciones si se cuenta con medios de comunicación no adecuados. Por otro lado, el $91 \%$ opinan que las negociaciones cara a cara presentan limitaciones de tiempo, espacio, número de personas que pueden participar en un lugar específico y fácilmente se pueden generar presiones hacia la otra parte y son ideales para negociaciones de alto nivel, alto costo, donde una decisión final es crucial. Adicional a esto, 95\% opinan que las negociaciones electrónicas fueron rápidas y muy útiles cuando la otra parte se encontraba en lugares muy lejanos, permitiéndoles realizar negociaciones en cualquier lugar, a cualquier hora, ahorrando costos de viajes, solucionando asuntos urgentes y $65 \%$ sienten que las negociaciones electrónicas requieren confianza entre dos o más partes a la hora de negociar, porque el elemento de la duda siempre estará presente cuando se realicen negociaciones a través de TIC.

\section{Metodología}

Haciendo uso de datos oficiales del año 2012 del Ministerio de Hacienda y Crédito Público de Colombia publicados por la Dirección de Impuestos y Aduanas Nacionales (DIAN) se han podido identificar entre diferentes empresas (exportadoras e importadoras) del país, como empresas de la ciudad de Medellín que han logrado internacionalizarse además que en efecto requieren llevar a cabo una negociación, a fin de cerrar todas sus operaciones de compraventa internacional, y que por supuesto no solo conocen las TIC sino que incluso en mayor o menor medida hacen uso de estas.

Tras esta caracterización el presente estudio indaga entonces sobre algunos de los motivos más comunes del porqué las TIC no son adoptadas en la totalidad de estas empresas-en su mayoría Pymes-en la negociación para los procesos de compraventa internacional, particularmente cuando parece existir una amplia bibliografía que tiende a demostrar no solo todas las ventajas que puede traer estas herramientas tecnológicas para optimizar los intercambios comerciales entre empresas, elevar la competitividad además de ofrecer una multiplicidad de alternativas en la identificación de clientes potenciales concretamente para pequeñas y medianas empresas (Jevtic, Dedjanski, Beslac, Grozdanic y Damnjanovic, 2013); además de los efectos positivos que tienen todas estas tecnologías no solo para la construcción de confianza sino también para una sucesiva materialización y mejores relaciones comerciales (Yamamoto, Tagami y Nakazawa, 2012).

En este sentido cabe destacar que las empresas objeto de esta investigación, se encuentran tanto dedicadas a la exportación como a la importación, y son tenidas en cuenta precisamente porque la negociación es de doble vía y porque evidentemente las operaciones de compraventa internacional tienden a favorecer no solo a las empresas que abastecen y distribuyen sus productos hacia otros mercados internacionales, sino también a las empresas que se abastecen de otras empresas proveedoras extranjeras.

En este estudio se tuvieron en cuenta 180 empresas de la ciudad de Medellín dedicadas a actividades de importación y/o exportación, las cuales fueron seleccionadas a través de un muestreo aleatorio estratificado y a quienes se les aplicó en el año 2013 una entrevista telefónica semiestructurada de carácter cualitativo. Con esto se buscó establecer una noción general respecto al patrón de conducta de las empresas a nivel general de la ciudad de Medellín, en torno al cómo estas llevan a cabo sus procesos de negociación y de qué manera las TIC influyen o no en este proceso desde su percepción.

En este sentido, una vez identificadas todas las empresas a estudiar en la ciudad de Medellín, se procedió a aplicar un instrumento de investigación (entrevista telefónica semiestructurada) seguido de un análisis cualitativo y descriptivo, que en primer lugar abordó las razones que impiden la implementación de TIC especializadas en negociación internacional. 
En segundo lugar, se estudió aspectos tales como las principales desventajas en el uso de las TIC, es decir, aquellos inconvenientes que estas empresas perciben sobre estas herramientas al momento de emplearlas dentro de la negociación en los procesos de compraventa internacional.

En tercer lugar el estudio, permitió detectar las preferencias en la manera de negociar de las empresas de la ciudad de Medellín, entre negociación cara a cara o negociación a través de las TIC; esto se hizo debido a que existen retos importantes que superar en el manejo de las relaciones comerciales, como el hecho que puede generar la despersonalización en el intercambio, como también la percepción misma que se tienen aún de la Internet como herramienta tecnológica desde la misma ética de la negociación (Yang, Chandlrees, Lin y Chao, 2009) entre otros asuntos propios a las transacciones en donde la identidad y la confianza entre las partes puede generar problemas en este sentido.

En cuarto lugar, se indagó sobre la percepción que tienen las empresas respecto al mejoramiento de las relaciones al utilizar las TIC para la negociación internacional, ya que existen indicios sobre los beneficios que las TIC generan a través del uso de la Internet y el intercambio de datos estandarizados con los socios comerciales a través de herramientas especializadas, las cuales contribuyen en aumentos significativos de productividad reflejados en la cadena de abastecimiento (Nurmilaakso, 2005).

Así es entonces, como la metodología del presente estudio se enfocó en establecer los cuatro puntos mencionados a partir de la percepción de empresas que se han internalizado vía operaciones de compraventa, específicamente a través exportación y/o importación

\section{Resultados y discusión}

Al caracterizar la muestra de las empresas se encontró que estas son en su mayoría Pymes y en cuyo caso sobresalen empresas del sector secundario con $52 \%$ dedicadas a la transformación de materias primas en bienes terminados, empresas del sector terciario con un $46 \%$ dedicadas la venta de servicios y empresas del sector primario con solo un $2 \%$ dedicadas a la extracción y generación de recursos con bajo nivel de transformación.

A continuación se presentan los resultados de los cuatro aspectos en los cuales se enfocó el instrumento de investigación mencionado en la metodología:

\subsection{Razones para no implementar las TIC especializadas en el proceso de negociación internacional}

Con base en la entrevista realizada a empresas importadoras y/o exportadoras, se logró identificar en la Tabla 2 las principales razones por las cuales estas empresas no utilizan las TIC especializadas en la negociación internacional, tales como software de gestión de proveedores (evaluación, clasificación y control de proveedores), plataformas virtuales (E-Procurement, E-Sourcing, E-Bidding), videoconferencia, sistemas soporte a la negociación (N..), software para la construcción de escenarios (teoría de juegos y probabilidades), y portales web especializados para la compraventa internacional.

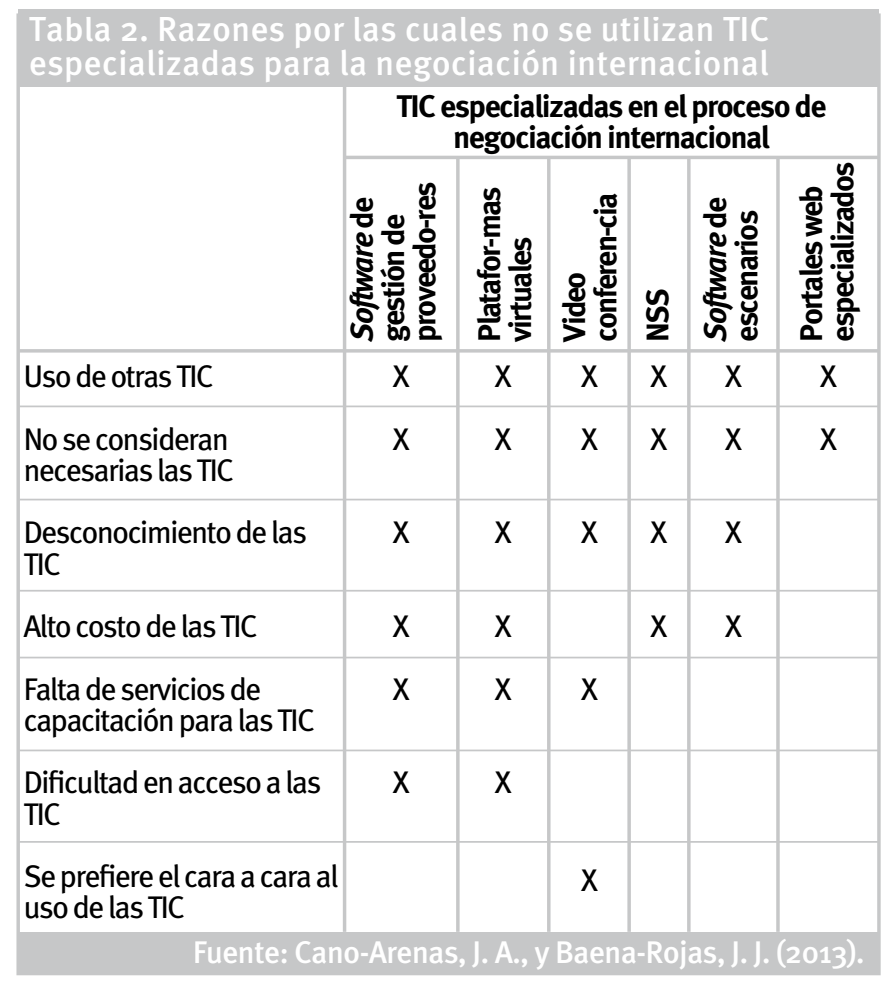

Para hacer la Tabla 2, se crearon categorías de las razones por las cuales estas empresas no utilizan las TIC especializadas (uso de otras TIC, no se considera necesaria las TIC, desconocimiento de las TIC, alto costo de las TIC, falta de servicios de capacitación para las TIC, dificultad en acceso a las TIC, se prefiere el cara a cara al uso de las TIC) y luego realizó un cruce entre las TIC especializadas y estas razones de no uso. De esta forma, cada celda de la Tabla 2 que tenga es su interior una $X$ representa que al menos un empresario de los entrevistados considera que una determinada TIC especializada no se utiliza debido a la razón que corresponde a la fila en la que está ubicada la celda en cuestión.

De acuerdo con la Tabla 2 se puede observar que existen dos razones transversales a todas las TIC especializadas que hacen que estas no se utilicen en las empresas para la negociación internacional; estas razones principales son que las empresas utilizan otras TIC en la negociación internacional (correo electrónico, mensajería instantánea, teléfono, entre otras) y que en las empresas no se tiene conciencia o necesidad de uso de las TIC para los procesos de compraventa que actualmente llevan a cabo. De forma similar, el desconocer 
la existencia o utilidad de las TIC especializadas en negociación internacional hacen que las empresas no se motiven a implementarlas en sus procesos, a excepción de los portales web especializados.

También una de las razones por las cuales no se implementan software de gestión de proveedores, plataformas virtuales, sistemas NSS y software para la construcción de escenarios, es el alto costo asociado con estas TIC que se debe al nivel de especialización y prestaciones que ofrecen para la negociación internacional.

La falta de servicios de capacitación para las TIC, la dificultad en acceso a las TIC y el preferir hacer negociaciones cara a cara son razones para el no uso de software de gestión de proveedores, plataformas virtuales y videoconferencia.

Estos resultados sugieren que en las empresas de exportación y/o importación se requiere crear conciencia y divulgar información sobre la existencia y utilidad de las TIC especializadas para la negociación internacional, y estimular el uso de TIC especializadas que complementan o en ocasiones incluyen las prestaciones de las TIC genéricas que hoy en día están siendo utilizadas por las empresas.

Es igualmente importante destacar que fue pequeña la proporción de los entrevistados que señalaron a la dificultad de acceso a la TIC y preferencias de negociar cara a cara como razones principales para no implementar las TIC especializadas en los procesos de negociación internacional, lo cual aclara que actualmente el problema de esta implementación no es por la falta de oferta, disponibilidad de las TIC o por falta de cultura de uso de las TIC genéricas, sino más bien por el desconocimiento de la existencia de tecnologías de vanguardia y de falta de reconocer necesidad de nuevas herramientas por parte de los empresarios.

\subsection{Principales desventajas asociadas con el uso de TIC en la negociación internacional}

Respecto a la importancia de las desventajas asociadas con el uso de TIC en el proceso de negociación internacional se analiza la Gráfica 1, que presenta el porcentaje de empresas que consideran quétan relevantes son las desventajas enunciadas. De esta forma se obtiene que los empresarios consideren que las desventajas más importantes asociadas con la implementación de las TIC en la negociación internacional son la necesidad de capacitación del personal, las necesidades de infraestructura (hardware, velocidad de redes, entre otros), el alto costo de las TIC, la despersonalización de la negociación y la dificultad de uso de las TIC.

En menor proporción los empresarios consideran que son importantes las desventajas de resistencia del cliente/proveedor a implementar las TIC y la resistencia por parte del personal de la empresa a implementarlas.

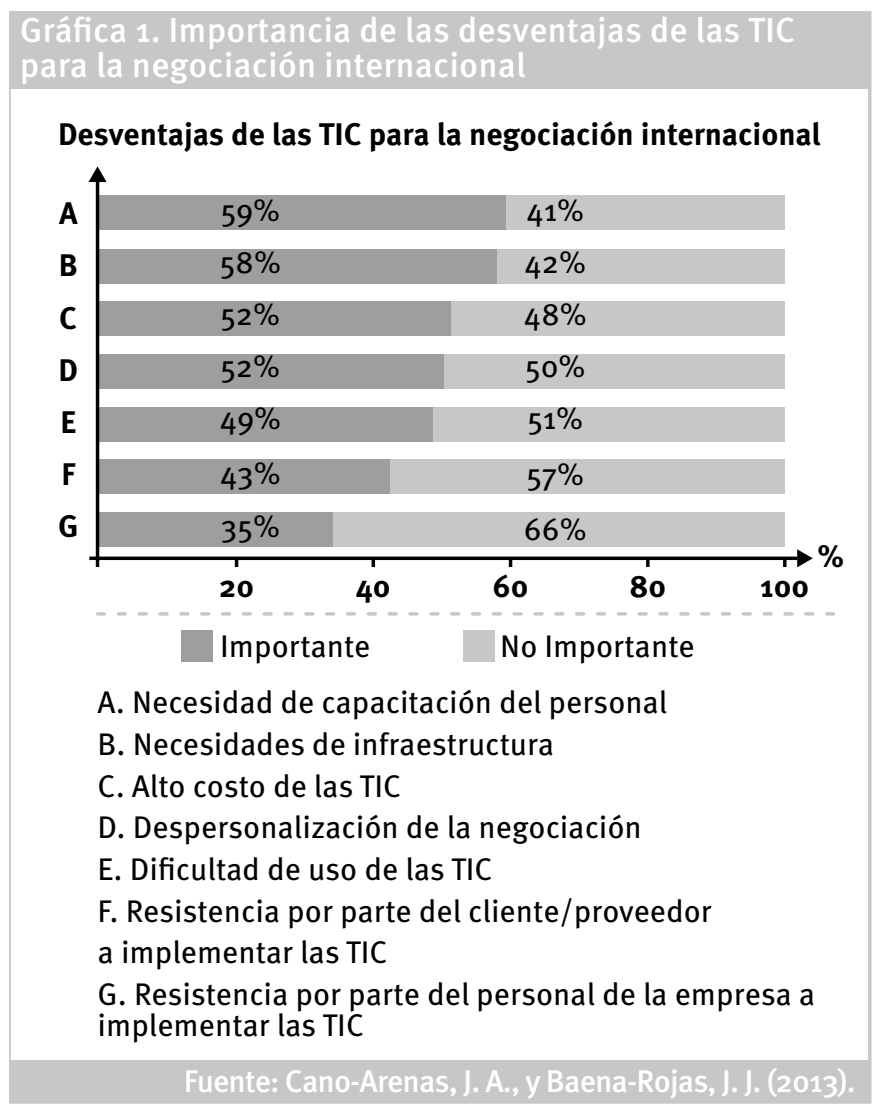

Esto permite mostrar que las principales desventajas percibidas en las empresas respecto a las TIC para la negociación internacional se deben en gran parte a la inversión a realizarse para que garantice un buen funcionamiento de estas, lo cual incluye capacitaciones, adaptaciones de tecnologías, servicios anexos, mientras que se perciben como menos importantes aquellas desventajas que se relacionan con la cultura al cambio tecnológico por parte de las personas que laboran en las empresas estudiadas como en los proveedores/clientes de estas empresas.

\subsection{Negociación cara a cara vs. negociación con TIC}

Para identificar los elementos que hacen que las empresas prefieran negociar cara a cara en lugar de negociar con intermediación de TIC, se observa el porcentaje de empresas que prefiere cada modo de negociación y luego se profundiza en las razones por las cuales los empresarios prefieren la negociación cara a cara. Es así como se observa entonces que en el $37,9 \%$ de las empresas se prefiere realizar negociaciones de compraventa internacional cara a cara, mientras que en un $62,1 \%$ prefiere que sean mediadas a través de las TIC, lo cual muestra una amplia aceptación de las TIC por parte de las empresas de Medellín para facilitar actividades dentro de la negociación internacional.

La Gráfica 2 muestra las razones por las cuales las empresasprefierenquesusnegociaciones decompraventa 
internacional se realicen cara a cara y el porcentaje de empresarios que acude a esta. Es así como se deduce que las principales razones para negociar cara a cara son el alcance de mayor efectividad en la negociación $(26,8 \%)$, la generación de más confianza y seguridad $(26,8 \%)$, la generación y mantenimiento de relaciones $(16,1 \%)$ y el apoyo y complemento al uso de TIC (12,5\%). En menor proporción, la personalización de la negociación $(5,4 \%)$ y el aumento de capacidad de persuasión (5,4\%): 7,1\% de las empresas realizan negociaciones cara a cara por múltiples razones que de manera individual no generan una participación significativa.

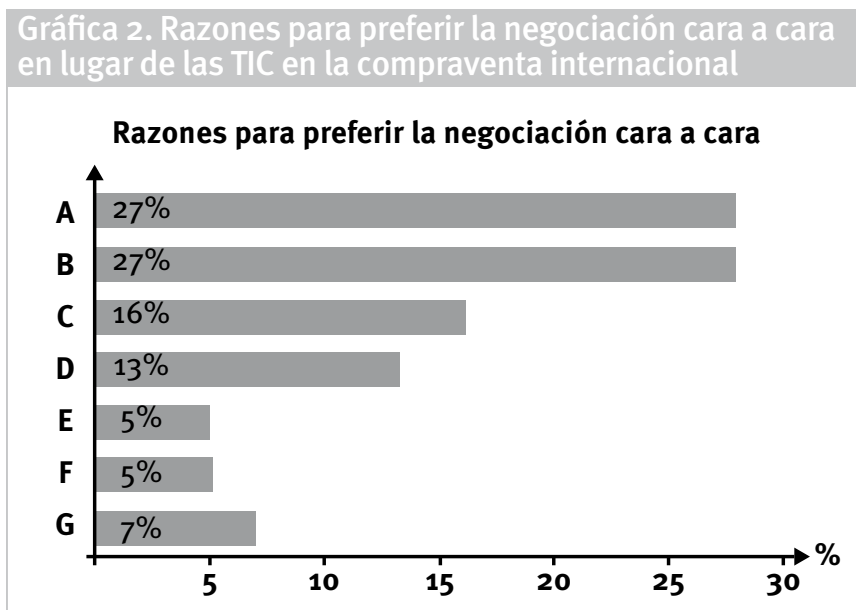
A. Permite ser más concreto y efectivo
B. Genera mayor confianza y seguridad
C. Genera y mantiene relaciones
D. Complementa el uso de TIC
E. Personaliza la negociación
F. Aumenta la capacidad de persuación
G. Otras razones

Fuente: Cano-Arenas, J. A., y Baena-Rojas, J. J. (2013).

Esta información revela que las empresas que prefieren negociar cara a cara lo hacen principalmente porque perciben que de esta manera se obtiene una mayor facilidad de alcanzar objetivos, se construyen mejores relaciones fundamentadas en la confianza, y se complementan actividades de negociación que se realizan con las TIC.

\subsection{Construcción de relaciones en la negociación con TIC}

En este estudio se hace necesario analizar cómo las TIC pueden contribuir a la construcción de relaciones en la negociación internacional, debido a que este es un aspecto fundamental para llevarlas con éxito. Para esto, se observa el porcentaje de empresas en las cuales se considera que las TIC en negociaciones internacionales mejoran las relaciones con sus clientes/proveedores, en comparación con los encuentros cara a cara.

La investigación muestra que en el 55,3\% de las empresas se considera que las TIC pueden contribuir a la construcción de relaciones en la negociación internacional, mientras que en un $44,7 \%$ considera que las negociaciones cara a cara contribuyen más al mejoramiento de relaciones en la negociación internacional. Esta información revela que para las empresas de exportación y/o importación de la ciudad de Medellín las TIC ayudan en mayor proporción a la creación de relaciones con clientes/proveedores que las negociaciones cara a cara.

A partir de esta información, en la Gráfica 3 se estudian las principales razones por las cuales las TIC mejoran las relaciones en negociaciones de compraventa internacional: las TIC facilitan el proceso de obtención y comunicación de información, ayudan a tener más claridad, ser más concisos, evitar malentendidos, tener soporte documentar de lo negociado $(28,3 \%)$, agilizan y aumentan la efectividad en la negociación permitiendo llegar más rápido a acuerdos, brindan menores tiempos de respuesta $(28,3 \%)$, reducen el tiempo, costo y distancias (17,2\%), aumentan la frecuencia de contacto ( $9,1 \%)$, y mantienen la relación con proveedores/ clientes luego de realizar un primer contacto con estos $(8,1 \%)$. En menor proporción, el evitar el contacto físico y visual $(6,1 \%)$ es también una razón por la cual las empresas consideran que las TIC contribuyen a mejorar relaciones. Un 3\% de las empresas consideran que existen otras razones adicionales a las mencionadas antes.
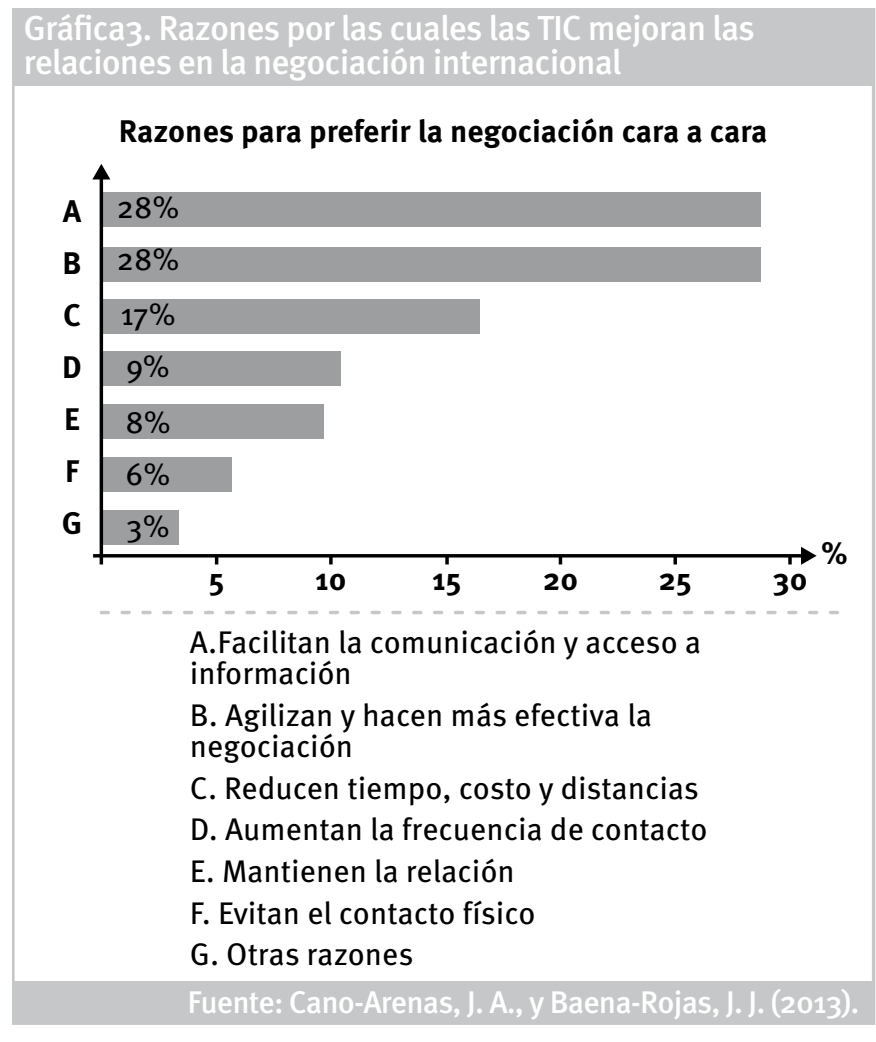

La información obtenida permite identificar que las TIC contribuyen a mejorar relaciones con clientes/proveedores al facilitar el contacto entre los negociadores porque brindan múltiples opciones de comunicación (visual, verbal, escrita o una combinación de las anteriores), permiten enfocarse en lo realmente 
importante de la negociación, ahorran desplazamientos y permiten que la comunicación se realice con mayor frecuencia.

\section{Conclusiones}

Las principales razones por las cuales las empresas no implementan las TIC especializadas para la negociación internacional no se limitan solo a los altos costos de estas tecnologías, sino también al uso actual de las TIC genéricas que cubren las necesidades básicas de las empresas, al desconocimiento de la existencia y utilidad de las TIC especializadas, y a la falta de reconocimiento por parte de las empresas de la necesidad de una actualización y especialización tecnológica para sus procesos de negociación internacional y encuentran que las principales desventajas que perciben las empresas respecto al uso en general de las TIC para la negociación internacional se relacionan con las inversiones en dinero y tiempo relativas a capacitaciones, infraestructura, adquisición en sí de las TIC.

Las TIC a utilizar por parte de las empresas para la negociación internacional tienen como reto permitir negociaciones de forma efectiva, generar confianza y un ambiente propicio para el mantenimiento de relaciones para que de esta forma reflejen los beneficios que actualmente tiene la negociación cara a cara, pero sin descuidar la facilidad de comunicación, agilidad para negociar, y la reducción de tiempo, costos y desplazamiento, que son las características que hacen que las TIC sean apreciadas y permitan mejorar las relaciones entre negociadores.

Se evidencia además que las empresas de Medellín son receptivas con las TIC para procesos de negociación internacional debido a que en su mayoría prefieren realizar negociaciones por estos medios, no consideran como desventaja importante la resistencia al cambio tecnológico por parte de sus empleados, proveedores y clientes, y reconocen múltiples ventajas que ofrecen las TIC, en especial para la construcción de relaciones. Sin embargo, se establece como reto utilizar en mayor proporción las TIC para la negociación internacional que sean especializadas y enfocadas en procesos de negociación internacional, y no utilizar para este fin herramientas genéricas que no permiten obtener máximos beneficios en la compraventa internacional.

Como futuros trabajos de investigación se sugiere realizar estudios que permitan identificar cuáles son las TIC más adecuadas para el proceso de negociación en las operaciones de compraventa internacional para una empresa de acuerdo con sus circunstancias y necesidades específicas, y de esta forma crear modelos que faciliten y orienten a las empresas a elegir correctamente las TIC para la negociación internacional.

De igual forma, se sugieren crear líneas de investigación que permitan relacionar la implementación de las TIC con el desempeño en los procesos de negociación internacional en cuanto a eficiencia y efectividad empresarial.

Finalmente, se invita a las empresas dedicadas al diseño y desarrollo de las TIC a estar receptivas con los resultados de esta investigación, e involucrar en las nuevas herramientas que se estén desarrollando todos aquellos aspectos que los empresarios aprecian de las TIC, y del mismo modo, implementar especificaciones en las TIC que permitan obtener los mismos beneficios que se están percibiendo por parte de los empresarios al negociar cara a cara.

\section{Referencias}

Albors-Garrigos, J., Hervas-Oliver, J., \& Marquez, P. (2009). Internet and mature industries. Its role in the creation of value in the supply chain. The case of tile ceramic manufacturers and distributors in Spain. International Journal of Information Management, 29 (6), 476-482.

Bayo-Moriones, A., Billon, M., \& Lera-Lopez, F. (2013). Perceived performance effects of ICT in manufacturing SMEs. Industrial Management y Data Systems, 113(1), 117-135.

Chai, S., \& Kim, M. (2012). A socio-technical approach to knowledge contribution behavior: An empirical investigation of social networking sites users. International Journal of Information Management, 32(2), 118-126.

Chang, Y. H., Chen, Y. T., Hung, M. H., \& Chang, A. Y. (2012). Development of an e-operation framework for sopc-based reconfigurable applications. International Journal of Innovative Computing Information and Contro, 8, 3639-3660.

Citera, M., Beauregard, R., \& Mitsuya, T. (2005). An experimental study of credibility in e-negotiations. Psychology y Marketing, 22 (2), 163-179.

Ebner, N., Bhappu, J., Gerarda, A., Brown, J., Kovach, K., \& Schneider, A. (2009). You've got agreement: negotiating via E-mail. En Honeyman, C., Coben, J., \& De Palo, G. (eds.). Rethinking negotiation teaching: innovations for ccontext and culture, (pp. 89-114). Milwaukee, United States: DRI Press.

Fernandez-Menendez, J., Lopez-Sanchez, J. I., Rodriguez-Duarte, A., \& Sandulli, F. (2009). Technical efficiency and use of information and communication technology in Spanish firms. Telecommunications Policy, 33 (7), 348-359.

Galin, A., Gross, M., \& Gosalker, G. (2007). E-negotiation versus face-to-face negotiation what has changed - if anything? Computers In Human Behavior, 23 (1), 787-797.

Hambrick, D. (2007). Upper echelons theory: An update. Academy of Management Review, 32 (2), 334-343.

Hatta, T., \& Ken-ichi, O. (2008). Effects of visual cue and spatial distance on exitability in electronic negotiation. Computers In Human Behavior, 24 (4), 1542-1551.

Jevtic, B., Dedjanski, S., Beslac, M., Grozdanic, R., \& Damnjanovic, A. (2013). SME technology capacity building for competitiveness and export - evidence from Balkan countries. Metalurgia International, 18 (4), 162-170.

Low, P. K., \& Ang, S. L. (2011). Information communication technology (ICT) for negotiations. Journal of Research in International Business and Management, 1 (6), 183-196.

Mibenge, M., \& Ngozika Chinyere, 0. (2007). E-procurement, internal customer service and external customers - a study of effects and interdependencies. (Tesis de Maestría). Blekin- 
ge Institute Of Technology, Sektionen för Management, Ronneby, Sweden.

Nurmilaakso, J. M. (2005). ICT solutions and labor productivity: evidence from firm-level data. Electronic Commerce Research, 9 (3), 173-181.

Pauleen, D., \& Yoong, P. (2001). Relationship building and the use of ICT in boundary-crossing virtual teams: a facilitator's perspective. Journal of Information Technology, 16 (4), 205-220.

Pennington, R., Wilcox, H., \& Grover, V. (2003). The role of system trust in business-to-consumer transactions. Journal of Management Information Systems, 20 (3), 197-226.

Sokolova, M., \& Szpakowicz, S. (2007). Strategies and language trends in learning success and failure of negotiation. Group Decision and Negotiation, 16 (5), 469-484.
Sokolova, M., Shah, M., \& Szpakowicz, S. (2006). Comparative analysis of text data in successful face-to-face and electronic negotiations. Group Decision and Negotiation, 15 (2), 127-140.

Thompson, L., \& Nadler, J. (2002). Negotiating via information technology: Theory and application. Journal of Social Issues, 58 (1), 109-124.

Yamamoto, T., Tagami, M., \& Nakazawa, M. (2012). A proposal for methodology for negotiation practicum with effective use of ICT - a technology enhanced course for communication for trust building. Turkish Online Journal of Educational Technology, 11 (4), 365-370.

Yang, M. H., Chandlrees, N., Lin, B., \& Chao, H. Y. (2009). The effect of perceived ethical performance of shopping websites on consumer trust. Journal of Computer Information Systems, 50 (1), 15-24.

Cuadernos de Administración / Facultad de Ciencias de la Administración / Universidad del Valle Periodicidad: semestral / ISSN impreso Nº 0120-4645-ISSN electrónico N² 2256-5078 / Nombre abreviado: cuad.adm. Edición Vol. $29 \mathrm{~N}^{\circ} 50$ (julio - diciembre de 2013)

Retos en la implementación de las TIC para el proceso de negociación internacional / José A. Cano Arenas y José J. Baena Rojas

\section{(c) (i) (3) (-)}

Revista Cuadernos de Administración por Universidad del Valle se encuentra bajo una Licencia Creative Commons Atribución-NoComercialCompartirlgual 2.5 Colombia.

Basada en una obra en http://cuadernosadm.univalle.edu.co. 